\title{
Epidemiological evidence for the fat theory
}

\section{By Flaminio Fidanza, Istituto di Scienza dell'Alimentazione, Università degli Studi, Perugia, Italy}

Evidence that dietary fats, among other factors, play a definite role in the aetiology of coronary heart disease (CHD) is partly based on epidemiological studies.

As a first approach, statistical information on death rates for arteriosclerotic heart disease (AHD) from the World Health Organization (WHO, 1955, 1965) can be correlated with dietary information from the food balance sheets published by the Food and Agriculture Organization (FAO, I949, 1966). Recently, Masironi (1970) made such a comparison for thirty-seven countries and found a strong positive correlation between AHD death rates and consumption of total fat and saturated fat. Intakes of other nutrients or food items showed lower positive or negative correlations or no correlation. A similar study (Stamler, Stamler \& Shekelle, I970) for twenty developed countries demonstrated a significant correlation between AHD death rates of middle-aged men and intakes of several nutrients: total energy, total fat, saturated fat, monounsaturated fat, cholesterol, total protein, animal protein, and sucrose, but not total carbohydrate or polyunsaturated fat. Of course this kind of correlative analysis can only give leads to possible cause-and-effect relationships, because - among other problems - many of these factors are intercorrelated with each other and with other features of the standard of living.

Cross-sectional epidemiological studies are also of limited value in assessing the relationship between the disease and causative factors, since they are only a single static 'look', based exclusively on disease prevalence. Mention will be made here only of the most relevant ones.

Some preliterate population groups with presumed high-fat intake have shown low serum cholesterol concentrations and low prevalence of CHD as evidenced by electrocardiograms. For the Masai it has been suggested (see Fidanza, 1970) that a genetic trait, producing an efficient feedback mechanism, is protecting them from hypercholesterolaemia. More recently, preliminary results of a metabolic study on Alaskan Eskimos suggest 'remarkable responses to dietary cholesterol' and this has been considered as 'the result of long-term adaptation to their customary high fat diet' (Taylor \& Ho, 1971). Further work is needed to verify the 'exceptional' nature of these populations, in terms of the dietary fat - serum cholesterol - AHD relationship, and to assess the validity of the genetic hypothesis concerning presumed interpopulation differences in cholesterol metabolism.

Valuable results have recently been published on 1372 Micronesian adults (Hankin, Reed, Labarthe, Nichaman \& Stallones, 1970) living in California, Guam 
and Rota of the Mariana Islands, and in Palau of the Caroline Islands. A dietary survey on about a third of the subjects showed a uniformity for the percentage of total energy from proteins, but the percentage from total fats decreased from about $40 \%$ in California to about $20 \%$ in Palau. The difference was particularly due to saturated fats. The percentage of total energy from carbohydrates showed a reverse pattern. Serum cholesterol and triglyceride concentrations, history of myocardial infarction, and ECG abnormalities were related to the differences in dietary intake of total and saturated fats.

According to some workers, prospective epidemiological studies should be considered of limited value because they can be useful in helping a community but not an individual. I doubt the validity of this criticism. After all, the entire practice of diagnostic and therapeutic medicine involves the application to individual patients of knowledge acquired from large series of cases. Obviously, for preventive efforts, as in diagnosis and treatment, individual differences must be recognized and considered. No one is proposing to apply epidemiological results dogmatically. But clinicians must appreciate that the epidemic of coronary disease, involving countries with hundreds of millions of people, cannot be brought under control through individual prevention. This is only a question of habit of mind. There is a difference in the approach to problems between the epidemiologist and the clinician. Both approaches are needed in complementary fashion to control the epidemic.

The main prospective epidemiological studies in which the lipid question has been considered in detail are the Pooling Project (see Stamler, 1971) and the International Cooperative Study on Epidemiology of Cardiovascular Disease Keys (1970).

In the Pooling Project the results were combined of the main prospective studies in the USA carried out in Albany, Chicago, Framingham, Los Angeles, and Minneapolis (see Stamler, I97I). Here the presentation is limited to the results obtained in the Framingham Diet Study (US Public Health Service, 1970); in general, the Pooling Project findings are similar. Significant results recently made available from Framingham (Kannel, 197I) demonstrated that 'serum lipids and cholesterol, in particular, are a prominent feature of the coronary profile and can be used to predict CHD and estimate risk. No lipid or battery of lipids appears more useful for this purpose than an accurate total serum cholesterol value in men or young women'. Excluding first those subjects with raised $\beta$-lipoprotein and then those with 'high' pre- $\beta$-lipoprotein values, the risk of CHD in the total population is in either instance still proportional to serum cholesterol concentration. The results also were consistent with the inference that the risk of CHD increases with raised endogenous serum triglycerides only if there is associated high serum cholesterol concentration. Of course this is true particularly for estimating the risk of CHD. The importance of lipoprotein pattern determination for diagnosis and therapy is another matter, requiring further elucidation.

The serum cholesterol concentration is influenced by diet. But in the Framingham study, as in the other studies in the USA where intakes of saturated fat and cholesterol were high, it was observed that the dietary components were not significantly 
related either to serum cholesterol concentration or to CHD incidence. Two main considerations have to be noted in this regard: at this high intake of nutrients such as saturated fat, cholesterol, and probably some carbohydrates, and bearing in mind the high proportion of total energy from fat, energy balance may become an important overriding factor. Then, and this is probably more important, in our International Cooperative Study (Keys, I970) we have shown that in dietary surveys repeated on the same individual, variation in the intake of the same nutrient is similar in magnitude to the variation between individuals. This means that within a relatively homogeneous population it is not possible to characterize with any certainty individual men in regard to nutrients in their diets. In fact, also in our study, we have not found significant correlations within cohorts between individual serum cholesterol values and the estimated nutrient intakes of those individuals. But the situation was very different when we compared contrasting population groups.

Our prospective epidemiological study on CHD started in 1958 with the collaboration of colleagues from Finland, Greece, Italy, Japan, The Netherlands, the USA, and Yugoslavia, with A. Keys as co-ordinator.

Sixteen 'chunk' samples of men aged $40-59$, resident for more than 5 years in a defined geographical area, were examined and followed for to years with quinquennial re-examinations. Thus we obtained results on both prevalence and incidence of the disease. The details for the standardized examination have been reported elscwhere (Keys, Aravanis, Blackburn, Buchem, Buzina, Djordjević, Dontas, Fidanza, Karvonen, Kimura, Lekos, Monti, Puddu \& Taylor, 1967). The 7-d individual weighing technique on a statistical subsample for each cohort, repeated in different seasons, was used for the dietary appraisal. For the USA railroad men the dietary interview and recall record were used. Further details can be found in an 'ad hoc' publication (Hartog, Buzina, Fidanza, Keys \& Roine, I968).

Because the Io year examinations are not yet completed for all the cohorts, we report here the results of the first 5 years (Keys, I970) limiting the cohorts to thirteen since dietary results for three of them are scanty.

The energy intake is, in general, high because most of the areas are rural, with a preponderance of farmers (Fidanza, 1972). The percentage of total energy from protein showed very little variation between populations, but this is not so for total energy from fats.

Before examining the relationship between some of the dietary results and incidence of CHD, let us note first that the correlation between one risk factor, serum cholesterol concentration, and the age standardized incidence rate of all CHD cases is 0.84 .

For dietary energy, expressed per $\mathrm{kg}$ body-weight, no correlation was found with incidence rate of all CHD cases $(r=0.04)$. The same was true for proteins expressed as percentage of total energy $(r=0.25)$. The results on percentage of total energy from fats plotted against the incidence rate of all CHD cases are presented in Fig. I. The correlation coefficient is low, $r=0.37$. But if we consider now only the saturated fatty acids, expressed as percentage of total energy, presenting the 


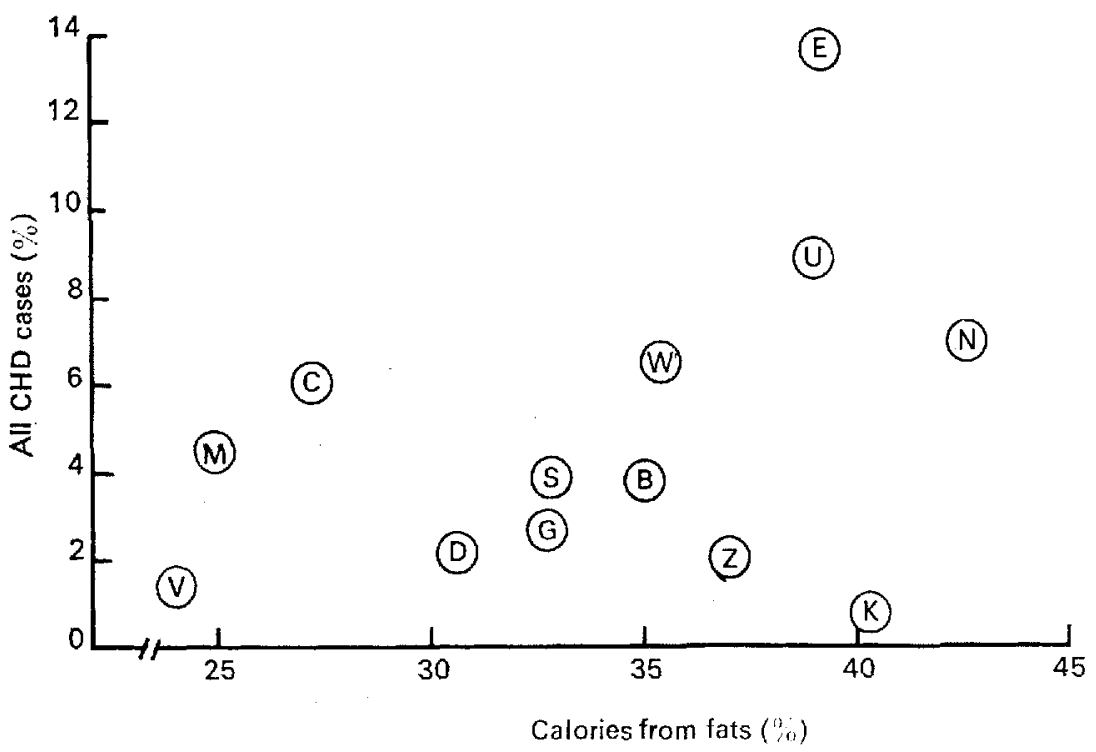

Fig. I. Five-year incidence rate of all $\mathrm{CHD}$ cases plotted against average percentage of total energy from fats in the diet in various cohorts $(r=0.37)$. B, Belgrade Faculty; C, Crevalcore; D, Dalmatia; E, East Finland; G, Corfu; K, Crete; M, Montegiorgio; N, Zutphen; S, Slavonia; U, USA Railroad; V, Velika Krsna; W, West Finland; Z, Zrenjanin.

main differences among cohorts, and plot them against incidence rate of all CHD cases (Fig. 2), we have a highly significant correlation coefficient, $r=0.86$ (Keys, 1970).

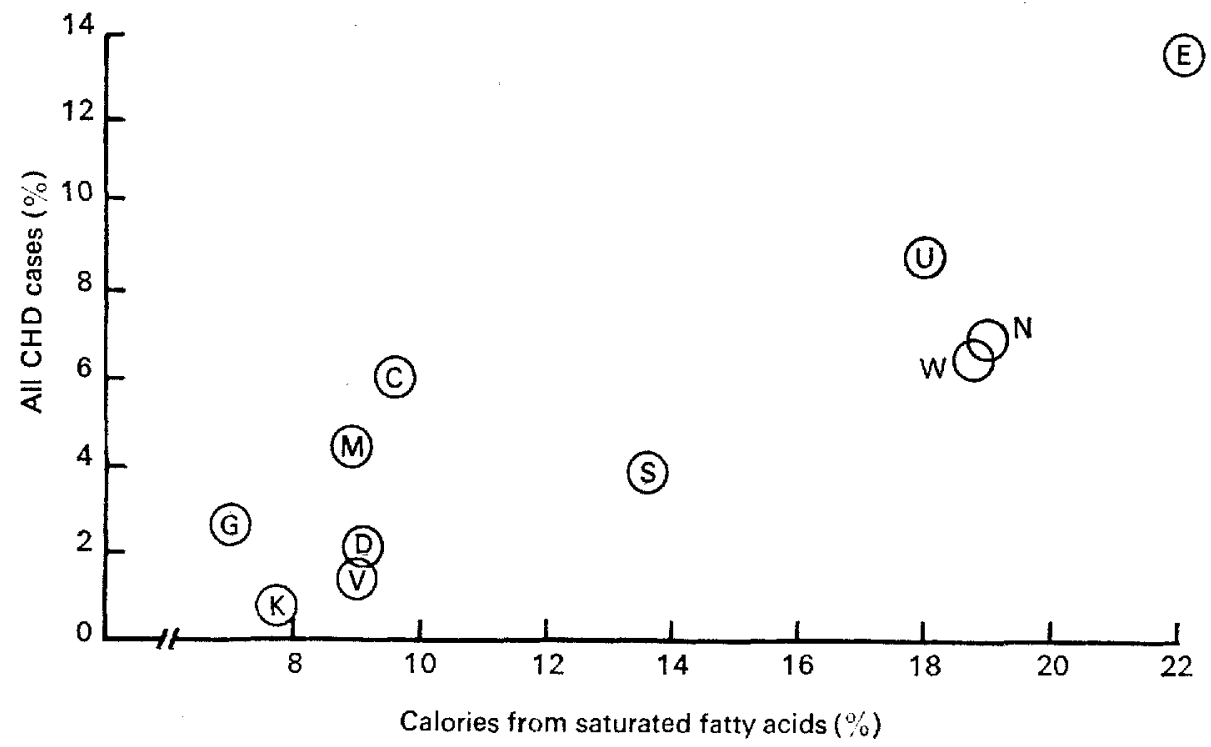

Fig. 2. Five-year incidence rate of all CHD cases plotted against average percentage of total energy from saturated fatty acids in the diet $(r=0.86)$. Cohort designations as in Fig. I. 
For mono-ene fatty acids expressed as percentage of total energy, a low negative correlation was found with the incidence rate of all CHD cases $(r=-0.40)$. The percentage of total energy from polyunsaturated fatty acids showed values very similar among the cohorts and no correlation with CHD incidence. But a high correlation was found between the expression $2 \mathrm{~S}-\mathrm{P}$ (where $\mathrm{S}=\%$ energy from saturated fatty acids and $\mathrm{P}=\%$ energy from polyunsaturated fatty acids) and the incidence rate of all CHD cases $(r=0.83)$.

The dietary cholesterol intake was computed for only a few cohorts, but we can correlate the percentage of total energy from eggs with the incidence rate of all CHD cases; the correlation coefficient for only nine cohorts was low $(r=0.25)$.

Other non-fat nutrients have shown a lower positive or negative correlation with CHD incidence. They have been considered in detail elsewhere (Fidanza, 1972). The results we obtained, together with those from studies on prevention of CHD, have been taken into consideration by both the Inter-Society Commission for Heart Disease Resources (1970) of the USA and, more recently, by the National Heart Foundation of Australia (1971) in making their recommendations.

We can conclude that the amount and the type of fats in the diet are important factors in the aetiology of the present epidemic of this disease. Also very important are combinations of risk factors, both from the epidemiological and preventive points of view.

\section{REFERENCES}

FAO (1949). Food Balance Sheets, 1947-48. Rome: Food and Agriculture Organization.

FAO (1966). Food Balance Sheets, r960-62. Rome: Food and Agriculture Organization.

Fidanza, F. (I970). Proc. int. Congr. Nutr. viII p. 5I. Amsterdam: Excerpta Medica.

Fidanza, F. (1972). Biblthca 'Nutr. Dieta'. (In the Press.)

Hankin, J., Reed, D., Labarthe, D., Nichaman, M. \& Stallones, R. (1970). Am. F. clin. Nutr. $23,346$.

Hartog, C. den, Buzina, R., Fidanza, F, Keys, A. \& Roine, P. (1968). Dietary Studies and Epidemiology of Heart Disease. The Hague: M. Wyt and Sons.

Inter-Society Commission for Heart Disease Resources (1970). Circulation 62, A55.

Kannel, W. B. (1971). Am. F. clin. Nutr. 24, 1074.

Keys, A. (editor) (1970). Circulation 4r, Suppl. no. I.

Keys, A., Aravanis, C., Blackburn, H. W., Buchem, F. S. P. van, Buzina, R., Djordjević, B. S., Dontas, A. S., Fidanza, F., Karvonen, M. J., Kimura, N., Lekos, D., Monti, M., Puddu, V. \& Taylor, H. L. (x967). Acta med. scand. 460, Suppl.

Masironi, R. (1970). Bull. Wld Hlth Org. 42, 103.

National Heart Foundation of Australia (1971). Med. F. Aust. I, 1155.

Stamler, J. (197r). Br. Heart F. 33, Suppl. p. 145.

Stamler, J., Stamler, R. \& Shekelle, R. B. (I97o). In Ischaemic Heart Disease p. 84 [J. H. de Haas, H. C. Hemker and H. A. Snellen, editors]. Leiden: Leiden University Press.

Taylor, C. B. \& Ho, K.-J. (1971). Am. F. clin. Nutr. 24, 129r.

US Public Health Service (1970). Publ. Hlth Monogr. no. 24.

WHO (1955). World Health Statistics Annual, 1955. Geneva: World Health Organization.

WHO (1965). World Health Statistics Annual, 1965. Geneva: World Health Organization. 